\title{
Enteral electrolytic solutions administered in continuous flow via naso-ruminal route in adult goats
}

\author{
Paulo Vinicius de M. SANTOS ${ }^{1) *}$, Rinaldo B. VIANA ${ }^{2)}$, \\ Marcel Ferreira B. AVANZA ${ }^{1)}$, Pedro Ancelmo N. ERMITA ${ }^{3)}$, Samuel R. ALVES ${ }^{1}$, \\ Micheline O. SILVA ${ }^{1}$, Lorena C. MONTEIRO ${ }^{1)}$, Caio M. COSTA ${ }^{1}$, \\ Erica G. MAFORT ${ }^{1}$, Lorraine Marcele L. COSTA $^{1)}$, Gabriella Maria M. FERREIRA ${ }^{1 \text { ), }}$ \\ Felipe S. MATTOS ${ }^{1)}$ and José D. RIBEIRO FILHO ${ }^{1)}$
${ }^{1)}$ Department of Veterinary, Federal University of Vicosa, Peter Henry Rolfs Avenue, w/n, University Campus, Viçosa, Minas Gerais, Brazil
2)Institute of Animal Health and Production, Federal Rural University of Amazonia, Presidente Tancredo Neves Avenue, 2501, Belém, Pará, Brazil
3)Institute of Studies of the Wet Tropic, Federal University of Southern and Southeastern Pará, Alberto Santos Dumont Street, w/n, Xinguara, Pará, Brazil

\section{J. Vet. Med. Sci. \\ 82(10): 1562-1569, 2020}

doi: 10.1292/jvms.20-0405

Received: 2 July 2020

Accepted: 10 August 2020

Advanced Epub:

31 August 2020

\begin{abstract}
This study aimed to investigate the effects of maintenance enteral electrolytic solutions administered naso-ruminally in continuous flow in adult goats subjected to water and food restriction. Six adult non-pregnant and non-lactating female goats, aged between two and five years old, were used in a crossover $(6 \times 2)$ study. Solution 1 (SEE1) comprised: $4.5 \mathrm{~g}$ sodium chloride $(\mathrm{NaCl}) ; 1 \mathrm{~g}$ potassium chloride $(\mathrm{KCl}) ; 0.5 \mathrm{~g}$ magnesium chloride $(\mathrm{MgCl}) ; 1 \mathrm{~g}$ calcium chloride in 1,000 m/ of water (measured osmolarity: $202 \mathrm{mOsm} / \mathrm{l}$ ). Solution 2 (SEE2) comprised: $4.5 \mathrm{~g}$ of NaCl; $1 \mathrm{~g}$ of KCl; $0.5 \mathrm{~g}$ of MgCl; $2 \mathrm{~g}$ of calcium acetate in 1,000 m/ of water (measured osmolarity: $212 \mathrm{mOsm} / \mathrm{l}$ ). The solutions were administered naso-ruminally at a dose rate of $15 \mathrm{~m} / /$ $\mathrm{kg} / \mathrm{hr}$, for $12 \mathrm{hr}$. The animals were evaluated at times T-24, T0, T4, T8, T12, and T24. Both enteral electrolytic solutions were effective in expanding blood volume. SEE1 showed a low-intensity acidifying potential, while SEE2 showed behavior of a neutral enteral electrolytic solution. KEY WORDS: acid-base balance, electrolyte replacement, enteral route, fluid therapy, hypotonic solutions
\end{abstract}

Among the metabolic disorders that affect adult ruminants, metabolic alkalosis stands out as the main acid-base disorder [26], which can be caused by prolonged periods of fasting and dehydration. Correction of this disorder can be achieved by several means, where the choice of the most effective treatment depends on the intensity of the condition the patient experiences.

Among the possibilities for correcting acid-base imbalances, enteral fluid therapy (EFT) is an effective option. In cases where there is no severe dehydration or hypovolemic shock, EFT has been shown to be as efficient as intravenous fluid therapy [8]. The advantages of EFT are easy applicability, possibility of modifying the composition of electrolytic solutions to the needs of each patient and its low cost.

However, similar to cattle, the administration of enteral electrolytic solutions in goats is performed via the oro-ruminal route in bolus. A disadvantage of bolus administration is abdominal distension, followed by discomfort, caused by the large volume of fluid deposited at once in the rumen $[21,23,24]$, in addition to the risk of lesions in the pharyngeal and esophageal mucosa caused by the successive applied of probes in this therapy [24].

In the EFT in continuous flow via naso-ruminal, the probe is introduced only once, remaining until the end of fluid therapy, in addition to the electrolyte solution being slowly infused in continuous flow, avoiding the possible problems that can occur when using the EFT in bolus. This technique has already been tested in experimental trials with several animal species [1, 12, 21, 23] and it has been successfully used in the clinical routine practiced by the authors of this manuscript for a number of years.

The main challenge in correcting hydroelectrolytic and acid-base imbalances in adult ruminants by an enteral modality remains the administration of electrolytic solutions that are effective and economically viable. Except for the study carried out by Atoji- 
Henrique et al. [2], there are no experimental tests with enteral electrolytic solutions in adult goats, which makes the present clinical trial relevant, since the composition of maintenance enteral electrolytic solutions for the species remains unknown. In addition, clinical trials with hypotonic enteral electrolytic solutions have not been performed in adult goats to date. However, considering the positive results of this type of solution in other animal species, it is assumed that they would also be satisfactory in adult goats.

Thus, the aim of this study was to investigate the effects of two maintenance hypotonic enteral electrolytic solutions administered via the naso-ruminal route in a continuous flow through a small-gage nasogastric tube for $12 \mathrm{hr}$ in adult goats subjected to water and food restriction. It was hypothesized that the use of the solution with a higher concentration of chloride and a strong ion difference (SID) equal to zero (SEE1), in addition to increasing blood volume, would have a low acidifying effect in animals.

\section{MATERIALS AND METHODS}

The experimental procedures were approved by the ethics committee in animal use of Universidade Federal de Viçosa (CEUA/ UFV process number 88/2018) following the guidelines of Brazilian legislation edited by the National Council of Animal.

\section{Study design}

The study was carried out at the Veterinary Hospital of the Universidade Federal de Viçosa, Brazil. Six healthy Saanen x Pardoalpine crossbred female dairy goats aged between two and five years, with a body weight between $52-72 \mathrm{~kg}$, non-pregnant and non-lactating, were used. During the study, the goats were kept in individual masonry bays, $2 \times 2 \mathrm{~m}\left(4 \mathrm{~m}^{2}\right)$ with a shavings bed and fed with hay, water, and a mineral supplement ad libitum, and a concentrate ( $2 \%$ of body weight) mixed with chopped elephant grass (Pennisetum purpureum), divided into two portions a day before and after water and food restriction. The animals were submitted to $24 \mathrm{hr}$ of water and food restriction before the beginning of the fluid therapy period.

The animals were randomly included in the experiment in a crossover design with six animals $\times$ two treatments $(6 \times 2)$, where each animal received all treatments with an interval of seven days between them. The treatments with maintenance hydroelectrolytic solution were named and composed of solution 1 (SEE1): $4.5 \mathrm{~g}$ sodium chloride; $1 \mathrm{~g}$ potassium chloride; $0.5 \mathrm{~g}$ magnesium chloride; $1 \mathrm{~g}$ calcium chloride in 1,000 ml of water (measured osmolarity: $202 \mathrm{mOsm} / \mathrm{l}$; SID=0); solution 2 (SEE2): $4.5 \mathrm{~g}$ of sodium chloride; $1 \mathrm{~g}$ of potassium chloride; $0.5 \mathrm{~g}$ of magnesium chloride; $2 \mathrm{~g}$ of calcium acetate in $1,000 \mathrm{~m} l$ of water (measured osmolarity: $212 \mathrm{mOsm} / \mathrm{l}$; SID=22.6). The solutions were administered naso-ruminally at a dose rate of $15 \mathrm{ml} / \mathrm{kg} / \mathrm{hr}$, for $12 \mathrm{hr}$.

Immediately before fluid therapy started, the animals were contained in trunks suitable for small ruminants and were probed naso-ruminally with a silicone, non-toxic PVC probe, $5 \times 7 \mathrm{~mm}$ thick and $1.5 \mathrm{~m}$ long (Fig. 1). The probe was attached to a halter and connected to the enteral fluid therapy system, a $20 l$ water gallon, with a spiral tube containing a flow regulator (Fig. 2).

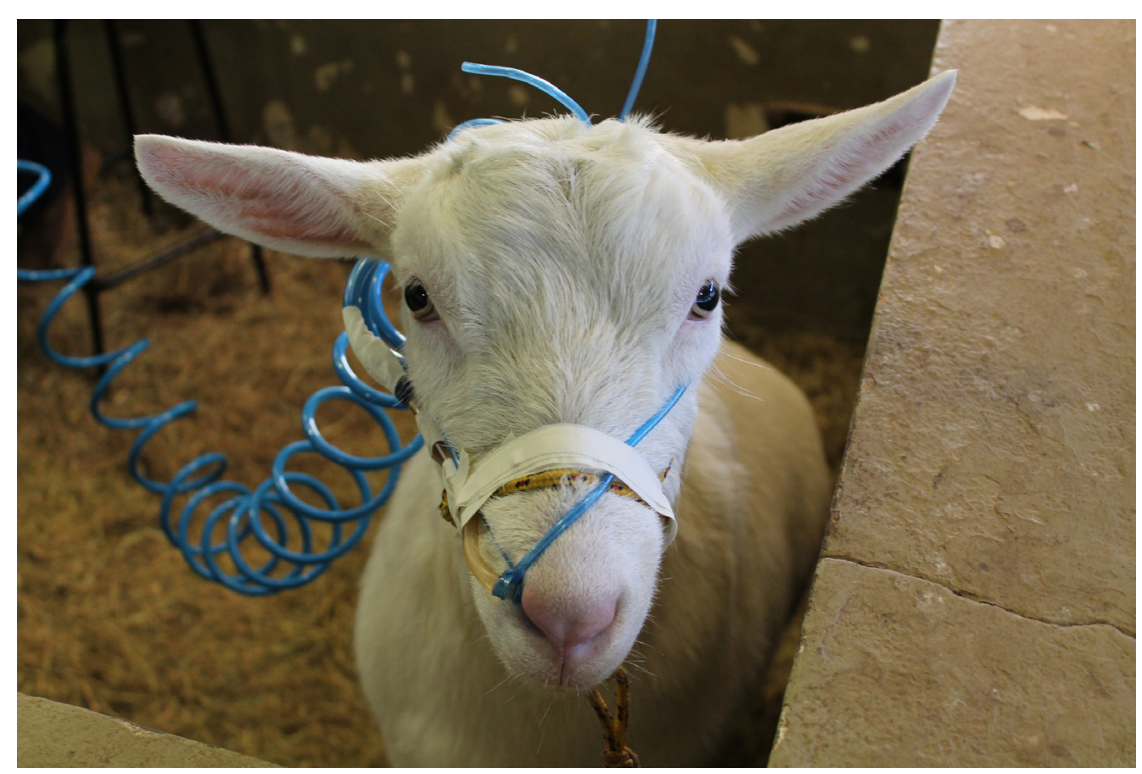

Fig. 1. Adult goat probed naso-ruminally with a silicone, non-toxic polyvinyl chloride probe, $5 \times 7 \mathrm{~mm}$ thick and $1.5 \mathrm{~m}$ long.

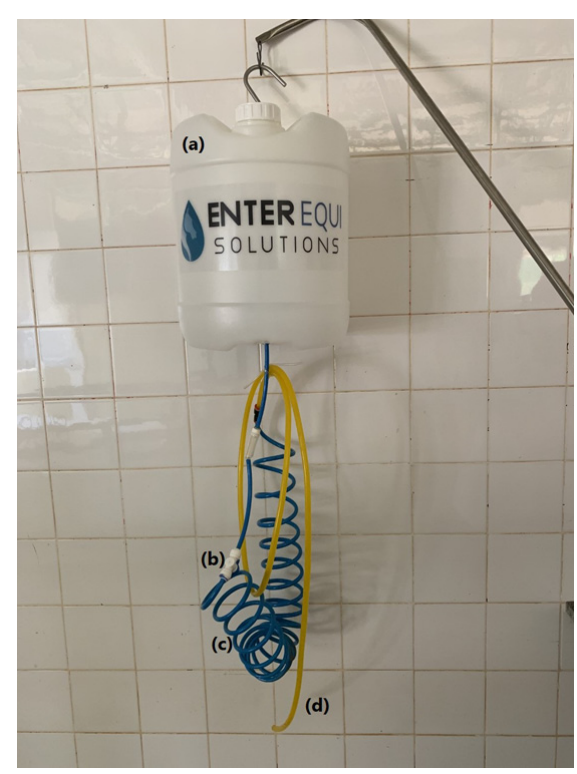

Fig. 2. Enteral fluid therapy system. $20 l$ water gallon (a), flow regulator (b), spiral tube (c) and probe (d). 


\section{Collection of biological samples and laboratory evaluations}

Biochemical, blood gas, and urinary analyzes were performed. The evaluations were carried out in the following moments: T-24: twenty four hr before the start of the fluid therapy phase, beginning of water and food restriction (control); T0: end of water and food restriction and the start of the fluid therapy phase; T4: four hr after fluid therapy started; T8: eight hr after fluid therapy started; T12: twelve hr of fluid therapy and end of the fluid therapy period; T24: twelve hr after the fluid therapy phase ended.

After antisepsis, blood samples were collected by jugular venipuncture, using a vacuum system for multiple collections with $25 \times 8$ mm (21G 1) needles (BD Vacutainer ${ }^{\circledR}$, Becton and Dickinson, São Paulo, Brazil). Blood was collected in vials (BD Vacutainer $^{\circledR}$, Becton and Dickinson) without anticoagulant to obtain serum, containing sodium fluoride to obtain plasma, and ethylenediamine tetraacetic acid (EDTA) to measure the packed cell volume (PCV) using the microhematocrit technique.

The samples were centrifuged and the serum and plasma aliquots frozen for further analysis. Serum osmolarity (Osm) was measured in an osmometer (model 3320, Advanced Instruments, Norwood, MA, USA) by the freezing point depression method. Serum elements: total plasma proteins (TPP), magnesium $\left(\mathrm{Mg}^{2+}\right)$, phosphorus (P) and creatinine (Creat) and plasma elements: glucose (Gluc) and lactate (Lac) were obtained with an automatic device HumaStar 300 (Human, In Vitro Diagnóstica Ltd., Itabira, Brazil) using a measurement kit (Bioclin-Quibasa Clínica Básica Ltd., Belo Horizonte, Brazil). In a selective ion analyzer (Humalyte plus 5, In Vitro Diagnóstica Ltd.) ionic calcium $\left(\mathrm{Ca}^{2+}\right)$, chloride $\left(\mathrm{Cl}^{-}\right)$, sodium $\left(\mathrm{Na}^{+}\right)$, potassium $\left(\mathrm{K}^{+}\right)$were measured in the serum.

For the blood gas evaluation, $2 \mathrm{~m} l$ of blood was collected in syringes (Vacuette, Centerlab Ltd., Belo Horizonte, Brazil) containing lithium heparin. The sample was processed using a blood gas analyzer (Radiometer ABL 5, Copenhagen, Denmark). The values of venous blood potential of hydrogen $(\mathrm{pH})$, partial pressure of carbon dioxide $\left(\mathrm{pCO}_{2}\right)$, bicarbonate concentration $\left(\mathrm{cHCO}_{3}{ }^{-}\right)$, concentration of titratable bases (BE) were determined, and the anion gap (AG) and strong ions difference (SID) were calculated according to the formulas proposed by Constable [6]:

$\mathrm{AG}=\left(\mathrm{Na}^{+}+\mathrm{K}^{+}\right)-\left(\mathrm{Cl}^{-}+\mathrm{HCO}_{3}^{-}\right)$

$\mathrm{SID}=\left(\mathrm{Na}^{+}+\mathrm{K}^{+}\right)-\left(\mathrm{Cl}^{-}\right)$

In the urine obtained by spontaneous urination at moments: $\mathrm{T}-24$ (samples collected in an interval of two hr), during the entire fluid therapy period, and at time T24 (samples collected in an interval of two hr), the total volume of the produced urine was measured, and biochemical analysis and filtered load (FL) calculations were made. Urine specific gravity (USG), performed by refractometry (Master-20T, Atago Brazil Ltd., São Paulo, Brazil), and urinary pH (upH), verified in a pH meter (DLA-PH, Del Lab, Araraquara, Brazil), were measured immediately after urine collection, and then an aliquot was taken to measure sodium (uNa ${ }^{+}$) and potassium $\left(\mathrm{uK}^{+}\right.$) by flame photometry (Photometry B462 Micronal, Santo Andre, Brazil), urinary creatinine (uCreat), chloride $\left(\mathrm{uCl}^{-}\right)$, total calcium $\left(\mathrm{uCa}^{2+}\right)$, and magnesium $\left(\mathrm{uMg}^{2+}\right)$ were made with an automatic device HumaStar 300 (Human, In Vitro Diagnóstica Ltd.) using a measurement kit (Bioclin-Quibasa Clínica Básica Ltd.).

The FL calculation was performed per animal using the urinary electrolyte $[\mathrm{uE}(\mathrm{mEq} / l)$ or $\mathrm{uE}(\mathrm{mg} / \mathrm{d} l)]$ value and multiplied by the volume of urine $[\mathrm{V}(l)$ or $\mathrm{V}(\mathrm{d} l)]$ produced in the same sample:

$\mathrm{uE}(\mathrm{mEq} / l) \times \mathrm{V}(l)=\mathrm{FL}(\mathrm{uE})$

$\mathrm{uE}(\mathrm{mg} / \mathrm{d} l) \times \mathrm{V}(\mathrm{d} l)=\mathrm{FL}(\mathrm{uE})$

Then, the results were grouped in time (GT) according to the serum collections. The entire FL (uE) calculation obtained during $\mathrm{T}-24$ and $\mathrm{T} 24$ was added and divided by two (duration in hr of collection) to determine a single result:

$[\mathrm{FL}(\mathrm{uE}) 1+\mathrm{FL}(\mathrm{uE}) 2+\mathrm{FL}(\mathrm{uE}) 3] \div 2=\mathrm{GTFL}(\mathrm{uE})$

The results obtained during the fluid therapy period (T0 to T12), were grouped into four stages so that a comparison could be made with the serum samples. For this, four periods of three hr were used: T0-3 (first three hr of fluid therapy); T3-6 (from the third hour of fluid therapy to the sixth hour of fluid therapy); T6-9 (from the sixth hour of fluid therapy to the ninth hour of fluid therapy); and T9-12 (from the ninth hour of fluid therapy to the end of fluid therapy). The FL (uE) obtained during these times were added in their proper grouping and divided by three (duration in hr of collection) with a single result being determined:

$[\mathrm{FL}(\mathrm{uE}) 1+\mathrm{FL}(\mathrm{uE}) 2+\mathrm{FL}(\mathrm{uE}) 3] \div 3=\mathrm{GTFL}(\mathrm{uE})$

\section{Statistical analysis}

Percentage data were submitted to the arc-sine transformation $\left(Y^{\prime}=\operatorname{arcsen} \sqrt{ } Y\right.$; t the other variables were subjected to the Kolmogorov-Smirnov and Bartlett tests, to verify the normality of errors and homogeneity of variances, respectively. When necessary, the variables were subjected to transformations according to the relationship between the means and variances [3] so that: the variables GTFL $\left(\mathrm{uNa}^{+}\right)$, GTFL $\left(\mathrm{uK}^{+}\right)$, GTFL $\left(\mathrm{uMg}^{2+}\right)$, GTFL $\left(\mathrm{uCl}^{-}\right)$, and GTFL (uCreat) were submitted to logarithmic transformation $\left(Y^{\prime}=\log (Y+1)\right)$; the variables $\mathrm{K}^{+}$, Osm, Gluc, and Lac were subjected to the root-square transformation $\left(Y^{\prime}=\sqrt{ } Y+0.5\right)$; and the USG variable was submitted to transformation $Y^{\prime}=Y-96$.

For analysis of the data, the Mixed Procedure of the Statistical Analysis System (SAS, version 9.4) was used. The experiment was conducted in a crossover $(6 \times 2)$ design with repeated measures over time, according to the model:

$Y i j k=u+S i+T j+(S T) i j+e i j k$

where: Yijk, observed response; $u$, constant; $S i$, effect of the solution; $T j$, effect of time; (ST) ij, interaction; eijk, random error.

The factor of repeated measures was the time (animal) for each level of animal (treatment) (subject) and a covariance matrix with a compound symmetry structure was used. The data were then submitted to analysis of variance and the means (LS-means) compared by $t$-test [18]. The level of significance adopted was $\alpha=0.05$. 


\section{RESULTS}

In the SEE1 animals, there was an increase $(P<0.05)$ in the PCV values at time T4 and in the TPP values at T0. In SEE1, Osm increased at T4, T8, and T12, while in SEE2 there was an increase only at T8 and T12. There was a difference between treatments at T8 (Table 1).

There was a significant increase in the amount of $\mathrm{Na}^{+}$in the SEE1 animals at T12 and in the SEE2 animals at T8 and T12. In turn, an increase in $\mathrm{K}^{+}$values was recorded in both treatments at $\mathrm{T} 12$. The values of $\mathrm{Cl}^{-}$showed similar behavior in the treatments, with an increase at T8, T12, and T24, however only in the SEE1 treatment was there a decrease at T0 (Table 2).

$\mathrm{Mg}^{2+}$ in SEE1 showed a significant decrease at T4 and T-24. In SEE2, the concentration of $\mathrm{Mg}^{2+}$ increased significantly at $\mathrm{T} 8$ and decreased $(P<0.05)$ at T24 (Table 2). A significant difference was observed between treatments at $\mathrm{T}-24$ and $\mathrm{T} 0$. Ca ${ }^{2+}$ values decreased at T8 and T12 in SEE1 and at T4, T8, and T12 in SEE2, while phosphorus values increased at T12 in SEE1 and at T8 in SEE2 (Table 2). Phosphorus values increased at T4 in both treatments and at T8 in SEE2 and at T12 in SEE1, at T24 both treatments decreased to their initial values. There were no significant changes $(P>0.05)$ for Gluc, Lac and Creat in the animals of the two treatments during the entire experimental phase (Table 2).

USG showed a significant reduction in its values in both treatments at T0-3, T6-9, and T9-12. The upH showed a difference between treatments at T24, while in SEE1, a decrease was recorded at T9-12 and T24. In SEE2, a decrease was detected at T24 (Table 3).

The variable uCreat increased at T0-3 and decreased at T3-6, T6-9, and T24 in SEE1 and increased at T0-3 and decreased at

Table 1. Mean \pm standard deviations for parameters of goats submitted to treatments with two enteral electrolytic solutions: Packed cell volume (PCV), Total plasma proteins (TPP) and Osmolarity (Osm)

\begin{tabular}{|c|c|c|c|c|c|c|c|}
\hline & \multirow{2}{*}{ Group } & \multirow{2}{*}{$\mathrm{T}-24$} & \multicolumn{4}{|c|}{ Fluid therapy phase } & \multirow{2}{*}{$\mathrm{T} 24$} \\
\hline & & & T0 & $\mathrm{T} 4$ & $\mathrm{~T} 8$ & T12 & \\
\hline PCV (\%) & SEE1 & $36.0 \pm 3.4^{\mathrm{cA})}$ & $38.8 \pm 2.7^{\mathrm{abA})}$ & $39.8 \pm 4.0^{\mathrm{aA})}$ & $37.2 \pm 1.9^{\mathrm{bcA})}$ & $35.2 \pm 2.6^{\mathrm{cA})}$ & $36.3 \pm 2.9 \mathrm{bcA})$ \\
\hline \multirow[t]{2}{*}{$\operatorname{TPP}(\mathrm{g} / \mathrm{d} l)$} & SEE1 & $6.9 \pm 0.3^{\mathrm{bcA})}$ & $7.3 \pm 0.4^{\mathrm{aA})}$ & $7.1 \pm 0.4^{\mathrm{abA})}$ & $6.9 \pm 0.3^{\mathrm{bcA})}$ & $6.7 \pm 0.3^{\mathrm{cA})}$ & $6.9 \pm 0.4 \mathrm{bcA})$ \\
\hline & SEE2 & $7.0 \pm 0.2^{\mathrm{abA})}$ & $7.2 \pm 0.4^{\mathrm{aA})}$ & $7.1 \pm 0.3^{\mathrm{abA})}$ & $6.9 \pm 0.3^{\mathrm{bA})}$ & $\left.6.8 \pm 0.3^{\mathrm{bA}}\right)$ & $6.9 \pm 0.4^{\mathrm{bA})}$ \\
\hline \multirow{2}{*}{$\begin{array}{l}\text { Osm } \\
(\mathrm{mOsm} / l)\end{array}$} & SEE1 & $297.5 \pm 1.0^{\mathrm{cA})}$ & $297.5 \pm 5.0 \mathrm{cA})$ & $300.5 \pm 3.4 \mathrm{bA})$ & $\left.302.2 \pm 7.1^{\mathrm{bB}}\right)$ & $306.8 \pm 5.0^{\mathrm{aA})}$ & $297.5 \pm 4.0^{\mathrm{cA})}$ \\
\hline & SEE2 & $\left.298.3 \pm 4.4^{\mathrm{bcA}}\right)$ & $296.8 \pm 2.8^{\mathrm{cA})}$ & $302.2 \pm 2.2^{\mathrm{bA})}$ & $309.2 \pm 4.7^{\mathrm{aA})}$ & $310.2 \pm 7.2^{\mathrm{aA})}$ & $299.8 \pm 3.7 \mathrm{bcA})$ \\
\hline
\end{tabular}

The mean values followed by different lowercase letters on the same line and different uppercase letters in the same column differ from each other $(P \leq 0.05)$.

Table 2. Mean \pm standard deviations for biochemical parameters of goats subjected to treatments with two enteral electrolytic solutions: Sodium $\left(\mathrm{Na}^{+}\right)$, Potassium $\left(\mathrm{K}^{+}\right)$, Chloride $\left(\mathrm{Cl}^{-}\right)$, Magnesium $\left(\mathrm{Mg}^{2+}\right)$, Calcium $\left(\mathrm{Ca}^{2+}\right)$, Phosphate $(\mathrm{P})$, Glucose (Gluc), Lactate (Lac) and Creatinine (Creat)

\begin{tabular}{|c|c|c|c|c|c|c|c|}
\hline & \multirow{2}{*}{ Group } & \multirow{2}{*}{$\mathrm{T}-24$} & \multicolumn{4}{|c|}{ Fluid therapy phase } & \multirow{2}{*}{$\mathrm{T} 24$} \\
\hline & & & T0 & $\mathrm{T} 4$ & $\mathrm{~T} 8$ & $\mathrm{~T} 12$ & \\
\hline \multirow[t]{2}{*}{$\mathrm{Na}^{+}(\mathrm{mmol} / \mathrm{l})$} & SEE1 & $141.1 \pm 1.5^{\mathrm{cA})}$ & $140.4 \pm 1.5^{\mathrm{cA})}$ & $142.5 \pm 1.8^{\mathrm{bcA})}$ & $144.4 \pm 2.7^{b A)}$ & $147.1 \pm 2.5^{\mathrm{aA})}$ & $143.8 \pm 1.4^{\mathrm{bA})}$ \\
\hline & SEE2 & $141.6 \pm 1.5^{\mathrm{bcA})}$ & $140.2 \pm 0.9^{\mathrm{cA})}$ & $143.1 \pm 1.5^{\mathrm{bA})}$ & $146.9 \pm 2.8^{\mathrm{aA})}$ & $148.5 \pm 4.0^{\mathrm{aA})}$ & $143.6 \pm 2.2^{\mathrm{bA})}$ \\
\hline \multirow[t]{2}{*}{$\mathrm{K}^{+}(\mathrm{mmol} / l)$} & SEE1 & $4.16 \pm 0.35^{\mathrm{cA})}$ & $3.84 \pm 0.25^{\mathrm{cA})}$ & $4.19 \pm 0.27^{\mathrm{bcA})}$ & $4.55 \pm 0.18^{\mathrm{abA})}$ & $4.72 \pm 0.23^{\mathrm{aA})}$ & $4.14 \pm 0.52^{\mathrm{cA})}$ \\
\hline & SEE2 & $4.11 \pm 0.40^{\mathrm{abA})}$ & $4.06 \pm 0.37^{\mathrm{bA})}$ & $4.23 \pm 0.11 \mathrm{abA})$ & $4.39 \pm 0.12^{\mathrm{abA})}$ & $4.48 \pm 0.36^{\mathrm{aA})}$ & $4.28 \pm 0.55 \mathrm{abA})$ \\
\hline \multirow[t]{2}{*}{$\mathrm{Cl}^{-}(\mathrm{mmol} / \mathrm{l})$} & SEE1 & $105.6 \pm 1.6^{\mathrm{cA})}$ & $103.0 \pm 1.0^{\mathrm{dA})}$ & $105.7 \pm 1.7^{\mathrm{cA})}$ & $109.4 \pm 2.0^{\mathrm{bB})}$ & $113.6 \pm 2.0^{\mathrm{aA})}$ & $110.8 \pm 1.0^{\mathrm{bA})}$ \\
\hline & SEE2 & $106.3 \pm 1.8^{\mathrm{cd} A)}$ & $104.5 \pm 2.0 \mathrm{dA})$ & $106.8 \pm 1.1 \mathrm{cA})$ & $112.1 \pm 2.2^{\mathrm{bA})}$ & $114.3 \pm 4.1 \mathrm{aA})$ & $110.5 \pm 2.5^{\mathrm{bA})}$ \\
\hline \multirow[t]{2}{*}{$\mathrm{Mg}^{2+}(\mathrm{mg} / \mathrm{d} l)$} & SEE1 & $2.88 \pm 0.24^{\mathrm{aA})}$ & $2.68 \pm 0.42^{\mathrm{abA})}$ & $2.57 \pm 0.28^{\mathrm{bA})}$ & $2.67 \pm 0.19 \mathrm{abA})$ & $2.70 \pm 0.11$ abA) & $2.48 \pm 0.34^{\mathrm{bA})}$ \\
\hline & SEE2 & $2.45 \pm 0.34 \mathrm{abcB})$ & $2.25 \pm 0.31^{\mathrm{cdB})}$ & $2.30 \pm 0.29 \mathrm{bcdA})$ & $2.55 \pm 0.18^{\mathrm{aA})}$ & $2.50 \pm 0.15^{\mathrm{abA})}$ & $2.20 \pm 0.37 \mathrm{dA})$ \\
\hline \multirow[t]{2}{*}{$\mathrm{Ca}^{2+}(\mathrm{mmol} / \mathrm{l})$} & SEE1 & $1.27 \pm 0.05 \mathrm{aA})$ & $1.24 \pm 0.07 \mathrm{abA})$ & $1.22 \pm 0.08^{\mathrm{bcA})}$ & $1.18 \pm 0.07^{\mathrm{cA})}$ & $1.20 \pm 0.04^{\mathrm{cA})}$ & $1.25 \pm 0.05 \mathrm{abA})$ \\
\hline & SEE2 & $1.23 \pm 0.06^{\mathrm{aA})}$ & $1.21 \pm 0.07 \mathrm{aA})$ & $1.17 \pm 0.09 \mathrm{bA})$ & $1.14 \pm 0.09 \mathrm{bA})$ & $1.17 \pm 0.08^{\mathrm{bA})}$ & $1.23 \pm 0.04 \mathrm{aA})$ \\
\hline \multirow[t]{2}{*}{$\mathrm{P}(\mathrm{mg} / \mathrm{d} l)$} & SEE1 & $4.82 \pm 0.53 \mathrm{dA})$ & $5.48 \pm 1.13^{\mathrm{cdA})}$ & $5.88 \pm 1.58^{\mathrm{bcA})}$ & $6.43 \pm 1.29^{\mathrm{abA})}$ & $7.08 \pm 0.71^{\mathrm{aA})}$ & $5.63 \pm 0.84^{b c d A)}$ \\
\hline & SEE2 & $4.70 \pm 1.26^{\mathrm{dA})}$ & $5.43 \pm 0.99 \mathrm{cdA})$ & $5.83 \pm 1.63 \mathrm{bcA})$ & $6.77 \pm 1.70^{\mathrm{aA})}$ & $6.58 \pm 1.49 \mathrm{abA})$ & $5.40 \pm 1.26^{\mathrm{cdA})}$ \\
\hline \multirow[t]{2}{*}{ Gluc (mg/d $l$ ) } & SEE1 & $61.8 \pm 6.5^{\mathrm{aA})}$ & $58.0 \pm 8.7^{\mathrm{aA})}$ & $61.0 \pm 7.6^{\mathrm{aA})}$ & $60.7 \pm 10.4^{\mathrm{aA})}$ & $62.5 \pm 9.5^{\mathrm{aA})}$ & $68.5 \pm 20.6^{\mathrm{aA})}$ \\
\hline & SEE2 & $58.2 \pm 12.5^{\mathrm{aA})}$ & $53.5 \pm 11.4 \mathrm{aA})$ & $62.2 \pm 13.6^{\mathrm{aA})}$ & $64.7 \pm 18.9$ aA) & $70.2 \pm 37.5^{\mathrm{aA})}$ & $56.2 \pm 19.8^{\mathrm{aA})}$ \\
\hline \multirow[t]{2}{*}{$\mathrm{Lac}(\mathrm{mg} / \mathrm{d} l)$} & SEE1 & $5.93 \pm 2.09^{\mathrm{aA})}$ & $6.77 \pm 6.19^{\mathrm{aA})}$ & $6.62 \pm 2.20^{\mathrm{aA})}$ & $6.00 \pm 1.17 \mathrm{aA})$ & $4.75 \pm 1.43^{\mathrm{aA})}$ & $6.72 \pm 7.26^{\mathrm{aA})}$ \\
\hline & SEE2 & $6.97 \pm 6.44^{\mathrm{aA})}$ & $\left.4.92 \pm 3.11^{\mathrm{aA}}\right)$ & $6.55 \pm 3.10^{\mathrm{aA})}$ & $7.10 \pm 5.78^{\mathrm{aA})}$ & $7.45 \pm 2.94^{\mathrm{aA})}$ & $5.88 \pm 3.59 \mathrm{aA})$ \\
\hline \multirow[t]{2}{*}{ Creat $(\mathrm{mg} / \mathrm{d} l)$} & SEE1 & $1.14 \pm 0.10^{\mathrm{aA})}$ & $1.20 \pm 0.26^{\mathrm{aA})}$ & $1.05 \pm 0.35^{\mathrm{aA})}$ & $1.10 \pm 0.31^{\mathrm{aA})}$ & $1.11 \pm 0.17^{\mathrm{aA})}$ & $1.20 \pm 0.16^{\mathrm{aA})}$ \\
\hline & SEE2 & $0.88 \pm 0.27 \mathrm{aA})$ & $1.15 \pm 0.23 \mathrm{aA})$ & $0.89 \pm 0.31 \mathrm{aA})$ & $0.90 \pm 0.18^{\mathrm{aA})}$ & $0.88 \pm 0.13^{\mathrm{aA})}$ & $\left.1.14 \pm 0.39^{\mathrm{aA}}\right)$ \\
\hline
\end{tabular}

The mean values followed by different lowercase letters on the same line and different uppercase letters in the same column differ from each other $(P \leq 0.05)$. 
Table 3. Mean \pm standard deviations for urinary parameters of goats submitted to treatments with two enteral electrolytic solutions: Urine specific gravity (USG), Urinary hydrogen potential (upH), Urinary creatinine (uCreat), Urinary sodium $\left(\mathrm{uNa}^{+}\right), \mathrm{Urinary} \mathrm{chloride}\left(\mathrm{uCl}^{-}\right)$, Urinary potassium $\left(\mathrm{uK}^{+}\right)$, Urinary calcium $\left(\mathrm{uCa}^{2+}\right)$ and Urinary magnesium $\left(\mathrm{uMg}^{2+}\right)$

\begin{tabular}{|c|c|c|c|c|c|c|c|}
\hline & \multirow{2}{*}{ Group } & \multirow{2}{*}{$\mathrm{T}-24$} & \multicolumn{4}{|c|}{ Fluid therapy phase } & \multirow{2}{*}{$\mathrm{T} 24$} \\
\hline & & & T0-3 & T3-6 & T6-9 & T9-12 & \\
\hline \multirow[t]{2}{*}{$\mathrm{USG}(\mathrm{g} / \mathrm{d} l)$} & SEE1 & $1,022.2 \pm 6.9 \mathrm{aA})$ & $1,019.5 \pm 6.0 \mathrm{abA})$ & $1,008.0 \pm 4.8^{\mathrm{cA})}$ & $1,004.4 \pm 2.8 \mathrm{dA})$ & $1,003.6 \pm 0.5 \mathrm{dA})$ & $1,014.4 \pm 5.6^{\mathrm{bA})}$ \\
\hline & SEE2 & $1,024.6 \pm 8.8 \mathrm{aA})$ & $1,018.0 \pm 7.0^{\mathrm{abA})}$ & $1,005.4 \pm 3.3^{\mathrm{cA})}$ & $1,003.2 \pm 1.0^{\mathrm{cA})}$ & $1,003.5 \pm 0.9^{\mathrm{cA})}$ & $1,013.8 \pm 3.0^{\mathrm{bA})}$ \\
\hline \multirow[t]{2}{*}{ upH } & SEE1 & $7.5 \pm 0.5^{\mathrm{aA})}$ & $7.4 \pm 0.4 \mathrm{aA})$ & $7.3 \pm 0.3^{\mathrm{abA})}$ & $7.0 \pm 0.3^{\mathrm{abA})}$ & $6.8 \pm 0.6^{\mathrm{bA})}$ & $4.9 \pm 0.6^{\mathrm{cB})}$ \\
\hline & SEE2 & $7.7 \pm 0.4 \mathrm{aA})$ & $7.1 \pm 0.7 \mathrm{aA})$ & $7.3 \pm 0.5^{\mathrm{aA})}$ & $7.2 \pm 0.3^{\mathrm{aA})}$ & $7.3 \pm 0.4 \mathrm{aA})$ & $6.2 \pm 1.0^{\mathrm{bA})}$ \\
\hline \multirow[t]{2}{*}{ uCreat $(\mathrm{mg} / \mathrm{d} l)$} & SEE1 & $75.6 \pm 30.6^{\mathrm{abA})}$ & $112.7 \pm 64.5^{\mathrm{aA})}$ & $47.9 \pm 12.4^{\mathrm{bA})}$ & $58.4 \pm 22.5^{\mathrm{bA})}$ & $72.0 \pm 22.5 \mathrm{abA})$ & $61.6 \pm 28.1 \mathrm{bA})$ \\
\hline & SEE2 & $48.2 \pm 22.2^{\mathrm{bA})}$ & $91.9 \pm 59.0^{\mathrm{aA})}$ & $46.3 \pm 16.1^{\mathrm{bA})}$ & $65.5 \pm 21.3^{\mathrm{abA})}$ & $48.7 \pm 13.9^{\mathrm{bA})}$ & $49.8 \pm 16.8^{\mathrm{bA})}$ \\
\hline \multirow[t]{2}{*}{$\mathrm{uNa}^{+}(\mathrm{mEq} / \mathrm{l})$} & SEE1 & $1.5 \pm 2.1 \mathrm{dA})$ & $1.7 \pm 1.7 \mathrm{dA})$ & $6.2 \pm 5.5^{\mathrm{cA})}$ & $20.9 \pm 9.1^{\mathrm{bA})}$ & $48.0 \pm 17.7^{\mathrm{aA})}$ & $21.2 \pm 6.9^{\mathrm{bA})}$ \\
\hline & SEE2 & $0.9 \pm 0.9 \mathrm{dA})$ & $2.0 \pm 2.3 \mathrm{dA})$ & $8.5 \pm 8.1^{\mathrm{cA})}$ & $34.1 \pm 11.8^{\mathrm{aA})}$ & $43.2 \pm 10.9^{\mathrm{aA})}$ & $16.5 \pm 9.5^{\mathrm{bA})}$ \\
\hline \multirow[t]{2}{*}{$\mathrm{uCl}^{-}(\mathrm{mg} / \mathrm{d} l)$} & SEE1 & $21.0 \pm 7.4 \mathrm{cA})$ & $5.8 \pm 4.6^{\mathrm{eA})}$ & $11.8 \pm 9.0^{\mathrm{dA})}$ & $37.8 \pm 22.4$ bcA) & $82.3 \pm 31.3^{\mathrm{aA})}$ & $56.3 \pm 20.2 \mathrm{abA})$ \\
\hline & SEE2 & $12.5 \pm 9.5^{\mathrm{bA})}$ & $6.4 \pm 4.7^{\mathrm{cA})}$ & $14.5 \pm 16.9^{\mathrm{bA})}$ & $59.2 \pm 29.0^{\mathrm{aA})}$ & $75.6 \pm 25.2^{\mathrm{aA})}$ & $51.9 \pm 22.7^{\mathrm{aA})}$ \\
\hline \multirow[t]{2}{*}{$\mathrm{uK}^{+}(\mathrm{mEq} / l)$} & SEE1 & $14.5 \pm 7.3^{\mathrm{aA})}$ & $5.0 \pm 1.9^{\mathrm{cA})}$ & $3.1 \pm 1.8^{\mathrm{cA})}$ & $4.6 \pm 1.8^{\mathrm{cA})}$ & $8.3 \pm 3.0^{\mathrm{bA})}$ & $9.9 \pm 7.1 \mathrm{abA})$ \\
\hline & SEE2 & $8.8 \pm 6.7^{\mathrm{abA})}$ & $3.0 \pm 2.2 \mathrm{cA})$ & $3.5 \pm 3.6^{\mathrm{cA})}$ & $5.1 \pm 2.4^{\mathrm{bA})}$ & $6.7 \pm 2.7 \mathrm{abA})$ & $9.4 \pm 5.2^{\mathrm{aA})}$ \\
\hline \multirow[t]{2}{*}{$\mathrm{uCa}^{2+}(\mathrm{mg} / \mathrm{d} l)$} & SEE1 & $39.5 \pm 40.8 \mathrm{abA})$ & $\left.27.6 \pm 30.5^{\mathrm{bA}}\right)$ & $\left.13.1 \pm 20.9^{\mathrm{bA}}\right)$ & $40.9 \pm 62.7$ abA) & $37.0 \pm 47.0 \mathrm{abA})$ & $\left.71.8 \pm 93.6^{\mathrm{aA}}\right)$ \\
\hline & SEE2 & $21.5 \pm 26.6^{\mathrm{aA})}$ & $28.2 \pm 40.2^{\mathrm{aA})}$ & $23.4 \pm 32.5^{\mathrm{aA})}$ & $34.0 \pm 32.3^{\mathrm{aA})}$ & $36.1 \pm 47.7 \mathrm{aA})$ & $56.0 \pm 44.1^{\mathrm{aA})}$ \\
\hline \multirow[t]{2}{*}{$\mathrm{uMg}^{2+}(\mathrm{mg} / \mathrm{d} l)$} & SEE1 & $34.9 \pm 19.0^{\mathrm{aA})}$ & $13.1 \pm 11.8^{\mathrm{bcA})}$ & $5.9 \pm 3.4^{\mathrm{cA})}$ & $11.0 \pm 3.4 \mathrm{bcA})$ & $15.9 \pm 4.3^{\mathrm{bA})}$ & $22.8 \pm 20.7^{\mathrm{abA})}$ \\
\hline & SEE2 & $\left.21.3 \pm 12.0^{\mathrm{aA}}\right)$ & $17.4 \pm 19.9^{\mathrm{abA})}$ & $8.5 \pm 7.5^{\mathrm{bA})}$ & $13.8 \pm 4.1^{\mathrm{abA})}$ & $16.3 \pm 4.2^{\mathrm{abA})}$ & $19.5 \pm 9.3^{\mathrm{aA})}$ \\
\hline
\end{tabular}

The mean values followed by different lower case letters on the same line and different upper case letters in the same column differ from each other $(P \leq 0.05)$.

Table 4. Mean \pm standard deviations for blood gas parameters of goats submitted to treatments with two enteral electrolytic solutions: Potential of hydrogen $(\mathrm{pH})$, Partial pressure of carbon dioxide concentration $\left(\mathrm{pCO}_{2}\right)$, Bicarbonate concentrations $\left(\mathrm{cHCO}_{3}^{-}\right)$, Bases excess $(\mathrm{BE})$, Anion gap (AG) and Strong ions difference (SID)

\begin{tabular}{|c|c|c|c|c|c|c|c|}
\hline & \multirow{2}{*}{ Group } & \multirow{2}{*}{$\mathrm{T}-24$} & \multicolumn{4}{|c|}{ Fluid therapy phase } & \multirow{2}{*}{$\mathrm{T} 24$} \\
\hline & & & T0 & $\mathrm{T} 4$ & $\mathrm{~T} 8$ & $\mathrm{~T} 12$ & \\
\hline \multirow[t]{2}{*}{$\mathrm{pH}$} & SEE1 & $7.41 \pm 0.02^{\mathrm{aA})}$ & $7.39 \pm 0.03^{\mathrm{abA})}$ & $7.37 \pm 0.02^{\mathrm{bcA})}$ & $7.35 \pm 0.02^{\mathrm{cd} A)}$ & $7.33 \pm 0.03 \mathrm{~dB})$ & $7.33 \pm 0.07 \mathrm{~dB})$ \\
\hline & SEE2 & $7.41 \pm 0.03 \mathrm{aA})$ & $7.38 \pm 0.05^{\mathrm{abA})}$ & $7.39 \pm 0.03^{a b A)}$ & $7.38 \pm 0.04 \mathrm{bA})$ & $7.39 \pm 0.04^{\mathrm{abA} A}$ & $7.39 \pm 0.03$ abA) \\
\hline \multirow[t]{2}{*}{$\mathrm{pCO}_{2}(\mathrm{mmHg})$} & SEE1 & $44.7 \pm 4.0^{\mathrm{abA})}$ & $47.9 \pm 4.5^{\mathrm{aA})}$ & $48.2 \pm 3.2^{\mathrm{aA})}$ & $46.3 \pm 3.8^{\mathrm{abA})}$ & $46.2 \pm 4.0^{\mathrm{abA})}$ & $43.7 \pm 4.2^{\mathrm{bA})}$ \\
\hline & SEE2 & $45.1 \pm 5.1 \mathrm{abA})$ & $48.2 \pm 8.0^{\mathrm{aA})}$ & $47.3 \pm 4.9 \mathrm{aA})$ & $46.3 \pm 5.8^{\mathrm{abA})}$ & $45.3 \pm 5.1 \mathrm{abA})$ & $43.4 \pm 3.7^{\mathrm{bA})}$ \\
\hline \multirow[t]{2}{*}{$\mathrm{cHCO}_{3}^{-}(\mathrm{mmol} / l)$} & SEE1 & $27.7 \pm 1.0^{\mathrm{abA})}$ & $28.0 \pm 1.1^{\mathrm{aA})}$ & $26.5 \pm 0.6^{\mathrm{bA})}$ & $24.4 \pm 1.0^{\mathrm{cB})}$ & $23.4 \pm 1.4^{\mathrm{cB})}$ & $21.8 \pm 1.7^{\mathrm{dB})}$ \\
\hline & SEE2 & $28.0 \pm 2.1 \mathrm{aA})$ & $27.6 \pm 1.1^{\mathrm{abA})}$ & $27.5 \pm 1.7 \mathrm{abA})$ & $26.3 \pm 1.3 \mathrm{bcA})$ & $\left.25.8 \pm 1.1^{\mathrm{cA}}\right)$ & $25.2 \pm 1.7^{\mathrm{cA})}$ \\
\hline \multirow[t]{2}{*}{$\mathrm{BE}(\mathrm{mmol} / l)$} & SEE1 & $3.15 \pm 0.78^{\mathrm{aA})}$ & $2.92 \pm 1.36^{\mathrm{aA})}$ & $1.65 \pm 0.54^{\mathrm{aA})}$ & $-0.45 \pm 1.03^{\mathrm{bA})}$ & $-1.98 \pm 1.54$ bcB) & $-3.17 \pm 3.25^{\mathrm{cB})}$ \\
\hline & SEE2 & $3.47 \pm 2.02^{\mathrm{aA})}$ & $2.45 \pm 1.27 \mathrm{abA})$ & $2.52 \pm 1.61^{\mathrm{aA})}$ & $0.83 \pm 1.13^{\mathrm{bA})}$ & $0.92 \pm 0.90 \mathrm{bA})$ & $1.07 \pm 2.10^{\mathrm{bA})}$ \\
\hline \multirow[t]{2}{*}{$\mathrm{AG}(\mathrm{mEq} / l)$} & SEE1 & $12.0 \pm 1.7^{\mathrm{cA})}$ & $13.3 \pm 1.8^{\mathrm{bcA})}$ & $14.5 \pm 1.1^{\mathrm{abA})}$ & $15.2 \pm 1.4^{\mathrm{aA})}$ & $14.8 \pm 1.4^{\mathrm{abA})}$ & $15.3 \pm 2.2^{\mathrm{aA})}$ \\
\hline & SEE2 & $11.3 \pm 2.4 \mathrm{aA})$ & $12.2 \pm 0.5^{\mathrm{aA})}$ & $13.0 \pm 1.9^{\mathrm{aA})}$ & $12.9 \pm 1.9 \mathrm{aB})$ & $12.9 \pm 1.7 \mathrm{aA})$ & $12.1 \pm 2.0 \mathrm{aB})$ \\
\hline \multirow[t]{2}{*}{$\mathrm{SID}(\mathrm{mEq} / l)$} & SEE1 & $39.7 \pm 1.0^{\mathrm{bA})}$ & $41.3 \pm 1.0^{\mathrm{aA})}$ & $41.0 \pm 1.3^{\mathrm{aA})}$ & $39.6 \pm 1.2^{\mathrm{bA})}$ & $38.1 \pm 1.2^{\mathrm{cA})}$ & $37.1 \pm 1.2^{\mathrm{cA})}$ \\
\hline & SEE2 & $39.4 \pm 1.5^{\mathrm{abA})}$ & $39.8 \pm 1.3^{\mathrm{abB})}$ & $40.5 \pm 1.5^{\mathrm{aA})}$ & $39.2 \pm 1.1^{\mathrm{bA})}$ & $38.7 \pm 1.2^{\mathrm{bA})}$ & $37.3 \pm 1.3^{\mathrm{cA})}$ \\
\hline
\end{tabular}

The mean values followed by different lowercase letters on the same line and different uppercase letters in the same column differ from each other $(P \leq 0.05)$.

T3-6, T9-12, and T24 in SEE2 (Table 3).

The concentrations of $\mathrm{uNa}^{+}$showed similar behavior in both treatments. The values increased from T3-6, remaining until $\mathrm{T} 24$. The $\mathrm{uK}^{+}$and $\mathrm{uCl}^{-}$showed similar behavior in both treatments, there was a reduction at T0-3 and an increase in their values from T6-9 to T24. The concentrations of $\mathrm{uCa}^{2+}$ decreased at T0-3 and T3-6 and increased at T24. The concentration of $\mathrm{uMg}^{2+}$ decreased at T0-3, T3-6, T6-9, and T9-12 in SEE1, while in SEE2 the decrease was observed only at T3-6 (Table 3).

There was a decrease $(P<0.05)$ in the $\mathrm{pH}$ values in SEE1 from T4 to T24. In SEE2, the decrease was recorded only at T8. In both treatments, $\mathrm{pCO}_{2}$ showed an increase $(P>0.05)$ at $\mathrm{T} 0$ and $\mathrm{T} 4$ in relation to $\mathrm{T} 24$. The values of $\mathrm{HCO}_{3}{ }^{-}$and $\mathrm{BE}$ showed similar behavior in both treatments. They decreased from T8 to T24. At those times, there was also a difference between treatments (Table 4).

The AG increased $(P<0.05)$ in SEE1 at T8 and T24, and showed a difference between treatments at T8 and T24 (Table 4). SID values increased in SEE1 at T0 and decreased at T24, while values in SEE2 increased at T4 and decreased at T12 and T24 (Table 4). 


\section{DISCUSSION}

Throughout the experiment, the animals remained alert, responsive, and active, showing no signs of discomfort or changes in attitude or behavior with the probe and the naso-ruminal probe for $12 \mathrm{hr}$, nor with the infusion of the enteral electrolyte solution at the dose of $15 \mathrm{~m} / \mathrm{kg}^{-1} \mathrm{hr}^{-1}$, in according to reports of Ribeiro Filho et al. [23] and Ribeiro Filho et al. [24]. It is noteworthy that this therapeutic modality has already been used in different animal species [1, 19, 21, 22]. Its use is a minimally stressful practice for animals, as described by other authors $[1,13]$.

The water and food restriction protocol caused a discreet change in some variables, such as PCV and TPP, demonstrating that 24 $\mathrm{hr}$ of water-food restriction is not enough to cause accentuated hydroelectrolytic and acid-base imbalances in this animal species, as the rumen functions as an important reservoir of water and goats are extremely adept at maintaining water balance and using the rumen as a water reserve for longer periods $[1,7,27]$.

Serum sodium and osmolarity did not show significant changes at $\mathrm{T} 0$, but increased in both treatments during the fluid therapy phase, reaching the highest values $12 \mathrm{hr}$ after the start of hydration $(P<0.05$; Tables 1 and 2$)$. This result demonstrates that, despite the hypotonicity of the enteral electrolyte solutions, the values of the variables mentioned above do not decrease, in contrast, there was an increase. Serum or plasma hyponatremia can occur when electrolyte solutions with low sodium are administered; this type of solution can also increase its intensity in patients who already have this type of electrolytic imbalance. However, the electrolytic solutions tested in the present study, by not having this type of adverse effect proved to be safe, and may even be used in patients with mild hyponatremia. Similar results to the present trial have been described in calves [21] and cattle [1].

Urinary sodium corroborates this statement, as during the fluid therapy period there was an increase in its excretion into the urine after three hr post-fluid therapy (T3-6), reaching the highest rates at $12 \mathrm{hr}$ of fluid therapy (T9-12) $(P<0.05$; Table 3$)$ and despite that, the animals did not present serum hyponatremia. It is noteworthy that this increase, in addition to the composition of the electrolytic solution, also had the contribution of decreasing aldosterone, because, with the increase in volume resulting from the action of enteral fluid therapy, there is a decrease in aldosterone, resulting in an increase in urinary sodium excretion [9]. However, as mentioned by Ribeiro Filho et al. [21], it should be emphasized that the use of hypotonic enteral electrolytic solutions should be done with caution, especially in cases of more pronounced hyponatremia, and with constant monitoring of the patient.

Serum potassium showed a slight change during the fluid therapy phase. This result demonstrates that electrolyte solutions were able to maintain potassium homeostasis in adult goats, confirming the results obtained by Atoji-Henrique et al. [2]. However, when evaluating the potassium excreted in the urine, it was noticed that from $\mathrm{T} 0$ there was a decrease in its values in animals in both treatments, maintaining this characteristic until the end of the fluid therapy phase. Aldosterone is the main modulator of potassium excretion in urine, increasing sodium absorption and potassium excretion [11]. With rehydration and increased blood volume in the animals, there was a decrease in aldosterone, with an increase in $\mathrm{uNa}^{+}$excretion and a decrease in $\mathrm{uK}^{+}$(Table 3). This decrease may also have been contributed to by the imposed fasting during the experimental phase ( $\mathrm{T}-24$ to $\mathrm{T} 12)$, because, as mentioned by Ward [30], the main source of potassium for ruminants is fodder. That said, in patients who are experiencing serum or plasma hypokalemia, as in diarrhea and metabolic alkalosis associated with lack of appetite or anorexia, the enteral electrolyte solution may contain more than $1 \mathrm{~g}$ of $\mathrm{KCl}$ per $l$.

The serum chloride showed a small decrease at $\mathrm{T} 0$ in the animals of both treatments. Then, there was a gradual increase over time, reaching the highest values at T12 in both treatments $(P<0.05$; Table 3$)$. The increase during the fluid therapy phase was similar to $\mathrm{Na}^{+}$, being related to the composition of the enteral electrolyte solutions. Both electrolytic solutions had salts that contained $\mathrm{Cl}^{-}$in their composition, thus, providing significant amounts of this electrolyte to the animals.

The animals of both treatments, at T12, presented a low serum chloride value above the reference range [15]. Although SEE1 contained more chloride in its composition $(111.1 \mathrm{mmol} / l)$ than SEE2 $(93.2 \mathrm{mmol} / l)$, unexpectedly, there was no difference between them at T12 ( $P>0.05$; Table 3). Despite the absence of a statistical difference, SEE1 promoted a $10.29 \%$ increase in serum chloride at the end of the hydration phase (T12), while in SEE2, the increase was 9.37\%. Perhaps the decrease in chloride in SEE2 may make it more suitable for use in prolonged treatment of animals that are not presenting hypochloremia.

As with urinary sodium excretion, urinary chloride excretion also increased. This increase occurs because most of the filtered chloride in the renal glomeruli is passively reabsorbed due to the reabsorption of sodium, therefore, because of the greater excretion of sodium there is also a greater excretion of chloride, justifying the findings of the present trial [29].

Serum $\mathrm{Ca}^{2+}$ and $\mathrm{Mg}^{2+}$ values showed a small decrease in SEE1 and SEE2 (Table 2). These decreases signal the possibility of an increase in $\mathrm{Ca}^{2+}$ and $\mathrm{Mg}^{2+}$ in the electrolyte solutions, since the urinary excretion of these two electrolytes also decreased, especially in animals treated with SEE1. When there is an increase in renal tubular flow, promoted by plasma expansion, there may be a decrease in the process of tubular reabsorption of some substances, among them calcium and magnesium [25], causing greater excretion of these elements. However, what was observed was a decrease in $\mathrm{uCa}^{2+}$ and $\mathrm{uMg}^{2+}$ concentrations. Therefore, these results point to the possibility of increasing the amount of $\mathrm{Ca}^{2+}$ and $\mathrm{Mg}^{2+}$ in the electrolytic solution, especially for patients who have serum or plasma hypocalcemia and hypomagnesemia.

The concentration of serum phosphorus increased in the fluid therapy phase in the animals of both treatments. Despite this increase, the values remained within the normal range mentioned by Jerry Kaneko et al. [15], making them without clinical significance.

Plasma glucose is not a better energy indicator in ruminants. They keep their blood concentrations low in relation to other species due to the use of fats and volatile fatty acids as an energy substrate [17]. It can be seen that during the whole experimental phase there were no changes in their values in both treatments $(P>0.05)$. Plasma lactate is a good indicator of cellular respiratory 
activity [14]. However, its behavior was similar to that of glucose; there were no significant changes $(P>0.05)$ in the animals of the two treatments during the entire experimental phase (Table 2).

Serum creatinine did not show significant changes during the entire experimental phase in animals from both treatments $(P>0.05)$. The urinary excretion of creatinine showed a small decrease during the fluid therapy period in both treatments. This decrease was caused by hemodilution resulting from the expansion of blood volume (Tables 2 and 3 ).

Urine specific gravity is considered a good marker of dehydration and volume expansion in animals. In the goats in the present trial, there was a significant decrease in USG in both treatments, reaching the lowest values at T9-12 $(P<0.05$, Table 3). The results of the present trial demonstrated the effective and mainly early effect of enteral fluid therapy in the expansion of blood volume, confirming the results obtained by Alves et al. [1], Dias et al. [8] and Monteiro et al. [19].

The blood $\mathrm{pH}$ of the animals that received SEE1 showed a slight decrease during the fluid therapy phase, reaching the lowest value at T12, maintaining the values at T24 $(P<0.05)$. In those that were infused with SEE2, the pH remained unchanged during the fluid therapy phase, remaining so until the moment of final evaluation. The decrease in $\mathrm{pH}$ from T8 observed in SEE1 animals reveals the presence of mild acidemia, which was maintained until the final $24 \mathrm{hr}$ of observation. Bicarbonate and cBase showed similar behavior to blood $\mathrm{pH}$, reaching the lowest values in SEE1 animals at T12 and T24. The cBase showed negative rates, $-3.7 \mathrm{mmol} / \mathrm{l}$ at T24, indicating the presence of mild metabolic acidosis (Table 4). The persistence of these changes at T24 was due to the volume of the electrolyte solution that remained in the rumen and that was absorbed after the end of the electrolyte solution administration (T12 to T24).

Solutions with SID less than $25 \mathrm{mEq} / \mathrm{l}$ have an acidifying potential [4]. The administration of this type of solution causes the appearance of metabolic acidosis, emphasizing that the intensity of the acidosis will depend on the amount of chloride, the volume, and the time of infusion of the solution. The composition of SEE1, presenting the same amount of cations and anions, that is, $\mathrm{SID}=$ zero, makes it acidifying. This characteristic translated into a decrease in the $\mathrm{pH}, \mathrm{cHCO}_{3}^{-}$, and cBase of the animals, confirming low-intensity metabolic acidosis. Electrolyte solutions with this peculiarity have a specific indication, patients with hypochloremic metabolic alkalosis. According to Roussel [26], more than 50\% of adult ruminants that arrive sick to hospitals for treatment have metabolic alkalosis, usually caused by changes in abomasal emptying causing hypochloremia. Electrolytic solutions such as SEE1 are indicated for the correction of these imbalances. Regarding SEE2, despite having caused a small decrease in cBase at T8, T12, and T24, the values remained in the normal range for goats [20].

The presence of mild metabolic acidosis caused by SEE1 was confirmed by the urinary pH of the animals at T9-12 and T24 $(P<0.05$, Table 3$)$. Goats feeding with a higher proportion of roughage have alkaline urinary pH like all herbivores [16]. The most intense aciduria recorded at T24 occurred $12 \mathrm{hr}$ after the end of hydration; it came from the effect of the residual volume of the electrolyte solution. The supply of the diet shortly after the end of fluid therapy may also have contributed to the decrease in urinary $\mathrm{pH}$ observed at $\mathrm{T} 24$. In turn, SEE2 did not change the urinary $\mathrm{pH}$ during the administration of the electrolytic solution, which registered a decrease in urinary $\mathrm{pH}$ only at $\mathrm{T} 24$, also signaling the contribution of the diet to this clinical observation.

A small increase in the $\mathrm{pCO}_{2}$ was recorded at T0 and T4 in the animals of both treatments (Table 4). However, despite this increase, the values remained within the normal range mentioned by Stevens et al. [28], who established the reference value for the variable in the caprine species between 34.6 and $48.8 \mathrm{mmHg}$.

Significant changes were observed in the AG values (Table 4) between treatments and in the SEE1 treatment over time $(P<0.05)$. The AG is used primarily to detect metabolic acidosis. This occurs when the values are above the reference range [10]. In SEE1 animals, the highest values of AG were detected at T8, T12, and T24, moments in which the lowest values of $\mathrm{cHCO}_{3}{ }^{-}$and $\mathrm{cBase}^{-}$ were recorded. In metabolic acidosis, $\mathrm{cHCO}_{3}{ }^{-}$and $\mathrm{cBase}$ decrease, as in the formula for calculating $\mathrm{AG}$ the value of $\mathrm{cHCO}_{3}{ }^{-}$is used. This decrease caused by its use to neutralize $\mathrm{H}^{+}$ions will be reflected in the increase in the value of AG, expressing the presence of metabolic acidosis generated by the composition of SEE1. In animals that received SEE2, there was no variation in the AG over time $(P>0.05)$. The difference between treatments occurred at T8 and T24, times when the AG of SEE1 was higher than SEE2 $(P<0.05)$, confirming the mild acidifying effect of SEE1.

The values for difference of strong ions showed no difference between the experimental groups $(P>0.05)$. At T8, T12, and T24, the lowest SID values were recorded in SEE1 and SEE2 (Table 4). This decrease was caused by chloride, since it occurred in the respective times that the highest values of the chloride ion were detected (Table 2). Care should be taken with the amount of chloride, because if the amount is exacerbated and the patient is not presenting hypochloremia, a type of acidosis that is common in animals, called hyperchloremic metabolic acidosis, can be produced iatrogenically [5], which has as a cause solutions with high chloride content or solutions with low SID. It is noteworthy that SEE1 caused the greatest decrease in SID.

In conclusion, the studied enteral electrolytic solutions are effective in expanding blood volume. As it contains more chloride and a lower SID, SEE1 demonstrated a low-intensity acidifying potential, which makes it a choice for patients with hypochloremic metabolic alkalosis, while SEE2 can be used as a maintenance electrolytic solution for patients with discrete or no electrolyte and acid-base imbalances.

ACKNOWLEDGMENTS. This work was supported by Fundação de Amparo à Pesquisa do Estado de Minas Gerais-FAPEMIG, Brazil (grant number: 5.273/15). The datasets generated for this study are available on request to the corresponding author. The authors have no competing or conflict of interests in submitting this article. 


\section{REFERENCES}

1. Alves, S. R., Avanza, M. F. B., Silva, M. O., Ermita, P. A. N., Gomes, L. L., Monteiro, L. C., Santos, P. V. M., Costa, C. M., Ferreira, G. M. M., Mattos, F. S. and Ribeiro Filho, J. D. 2019. Two enteral solutions with different chloride concentrations administered by naso-ruminal route for fluid therapy in adult cattle. Cienc. Rural 49: e20181011. [CrossRef]

2. Atoji-Henrique, K., Filho, J. D. R. and Malafaia, P. 2012. Enteral fluid therapy through nasogastric tube in rumen cannulated goats. Pesqui. Vet. Bras. 32: 1281-1284. [CrossRef]

3. Barbosa, J. C. O. 1983. [Heteroscedasticity problem in the analysis of variance]. p. 14. In: Seminar Presented to the ESALQ-USP Statistics and Experimentation Census, ESALQ, Piracicaba (in Portuguese).

4. Constable, P. D. 2014. Acid-base assessment: when and how to apply the Henderson-Hasselbalch equation and strong ion difference theory. Vet. Clin. North Am. Food Anim. Pract. 30: 295-316, v. [Medline] [CrossRef]

5. Constable, P. 2003. Fluid and electrolyte therapy in ruminants. Vet. Clin. North Am. Food Anim. Pract. 19: 557-597. [Medline] [CrossRef]

6. Constable, P. D. 2000. Clinical assessment of acid-base status: comparison of the Henderson-Hasselbalch and strong ion approaches. Vet. Clin. Pathol. 29: 115-128. [Medline] [CrossRef]

7. Dahlborn, K. and Karlberg, B. E. 1986. Fluid balance during food deprivation and after intraruminal loads of water or isotonic saline in lactating and anoestral goats. Q. J. Exp. Physiol. 71: 223-233. [Medline] [CrossRef]

8. Dias, D. C. R., Moreira, N. S., Bittencourt, T. B. S. C., Oliveira, D. P., Ermita, P. A. N., Silva, M. O., Fonseca, L. A. and Ribeiro Filho, J. D. 2019. [Comparative evaluation between different kinds of hydroeletrolitic reposition in horses experimentally dehydrated by the use of physical examination parameters and hematological markers]. Rev. Bras. Cienc. Vet. 26: 34-41 (in Portuguese).

9. DiBartola, S. P. 2012. Disorders of sodium and water: hypernatremia and hyponatremia. pp. 45-79. In: Fluid, Electrolyte, and Acid-Base Disorders in Small Animal Practice, 4th ed. (DiBartola, S. P. ed.), Elsevier Saunders, St. Louis.

10. DiBartola, S. P. 2012b. Introduction to acid-base disorders. pp. 231-252. In: Fluid, Electrolyte, and Acid-Base Disorders in Small Animal Practice, 4th ed. (DiBartola, S. P. ed.), Elsevier Saunders, St. Louis.

11. DiBartola, S. P. and De Morais, H. A. 2012. Disorders of potassium: hypokalemia and hyperkalemia. pp. 92-119. In: Fluid, Electrolyte, and AcidBase Disorders in Small Animal Practice, 4th ed. (DiBartola, S. P. ed.), Elsevier Saunders, St. Louis.

12. Ermita, P. A. N., Viana, R. B., Ribeiro Filho, J. D., Guimarães, J. D., Dias, D. C. R., Monteiro, B., Monteiro, L. C. and Moreira, A. R. P. 2016. Effects of enteral fluid therapy in continuous flow dministered by nasogastric tube in buffalo calves. Cienc. Rural 5: 60-69.

13. Gomes, C. L. N., Ribeiro Filho, J. D., Farias, S. K. and Donner, A. C. 2014. Effects of PEG 3350 or an enteral solution associated or not with Ringer lactate, and of $\mathrm{NaCl} 0.9 \%$ on the glucose, lactate, cortisol and insulin of healthy equines. Arq. Bras. Med. Vet. Zootec. 66: 1039-1045. [CrossRef]

14. Jerry Kaneko, J. 2008. Carbohydrate metabolism and its diseases. pp. 45-80. In: Clinical Biochemistry of Domestic Animals, 6th ed. (Kaneko, J., Harvey, J. W. and Bruss, M. L. eds.), Academics Press, San Diego.

15. Jerry Kaneko, J., Harvey, J. W. and Bruss, M. L. 2008. Appendix VIII blood analyte reference values in large animals. pp. 882-888. In: Clinical Biochemistry of Domestic Animals, 6th ed. (Kaneko, J., Harvey, J. W. and Bruss, M. L. eds.), Academics Press, San Diego.

16. Jones, M., Miesner, M. D., Baird, A. N. and Pugh, D. G. 2012. Diseases of the Urinary System. Pp. 325-360. In: Sheep and Goat Medicine, 2th ed. (Pugh, D. G. and Baird, A. N. eds.), Elsevier Saunders, Maryland Heights.

17. Kozloski, G. V. 2011. Bioquímica dos Ruminantes, 3rd ed. Editora da UFSM, Santa Maria.

18. Littell, R. C., Milliken, G. A., Stroup, W. W., Wolfinfer, R. D. and Schabenberger, O. 2006. SAS ${ }^{\circledR}$ for mixed models. 2nd ed. SAS Institute Inc., Cary.

19. Monteiro, L. C., Viana, R. B., Avanza, M. F. B., Ermita, P. A. N., Costa, C. M., Alves, S. R., Santos, P. V. M., da Silva, M. O., Balbino, D. A. B., de Mattos, F. S., Teixeira, R. B. C. and Ribeiro Filho, J. D. 2020. Effects of hypotonic and isotonic enteral electrolyte solutions administered in continuous flow in weaned foals. Front. Vet. Sci. 7: 280. [Medline] [CrossRef]

20. Redlberger, S., Fischer, S., Köhler, H., Diller, R. and Reinhold, P. 2017. Age-dependent physiological dynamics in acid-base balance, electrolytes, and blood metabolites in growing goats. Vet. J. 229: 45-52. [Medline] [CrossRef]

21. Ribeiro Filho, J. D., Dantas, W. M. F., Lima, A. P., Viana, R. B., Silva, M. O., Ermita, P. A. N., Silva, G. M. M., Ribeiro, B. M., Costa, C. M. and Monteiro, L. C. 2017. Enteral electrolyte solutions with different osmolarities administered in a continuous flow in newborn calves. Cienc. Rural 47: 1-6. [CrossRef]

22. Ribeiro Filho, J. D., Farias, S. K., Fonseca, L. A., Avanza, M. F. B., Dantas, W. M. F., Dias, D. C. R., Monteiro, L. C., Ribeiro, B. M. and Dantas, F. T. D. R. 2015. Enteral electrolyte solutions with different osmolarities: clinical and laboratory assessment in equines. J. Equine Vet. Sci. 35 : 673-678. [CrossRef]

23. Ribeiro Filho, J. D., Gimenes, A. M., Fonseca, E. F., Dantas, W. M. F. and Oliveira, T. T. 2011. Enteral fluid therapy in cattle: evaluation of isotonic electrolyte solutions administered via nasogastric tube on continuous flow. Cienc. Rural 41: 285-290. [CrossRef]

24. Ribeiro Filho, J. D., Avanza, M. F. B., Baptista Filho, L. C. F., Dantas, W. D. M. F., Lima, A. P. and Gomes, C. L. N. 2013. [Evaluation of isotonic electrolyte solution administered by enteral via in healthy cattle or dehydrated experimentally]. Vet. Zootec. 20: 9-16 (in Portuguese).

25. Riella, M. C. 2012. [Principles of Nephrology and Hydroelectrolytic Disorders], 5th ed. Guanabara Koogan, Rio de Janeiro (in Portuguese).

26. Roussel, A. J. 2014. Fluid therapy in mature cattle. Vet. Clin. North Am. Food Anim. Pract. 30: 429-439, vi-vii. [Medline] [CrossRef]

27. Silanikove, N. 2000. The physiological basis of adaptation in goats to harsh environments. Small Rumin. Res. 35: 181-193. [CrossRef]

28. Stevens, J. B., Anderson, K. L., Correa, M. T., Stewart, T. and Braselton, W. E. Jr. 1994. Hematologic, blood gas, blood chemistry and serum mineral values for a sample of clinically healthy adult goats. Vet. Clin. Pathol. 23: 19-24. [Medline] [CrossRef]

29. Waldrop, J. E. 2008. Urinary electrolytes, solutes, and osmolality. Vet. Clin. North Am. Small Anim. Pract. 38: 503-512, ix. [Medline] [CrossRef]

30. Ward, G. M. 1966. Potassium Metabolism of Domestic Ruminants -A Review. J. Dairy Sci. 49: 268-276. [CrossRef] 